\title{
Scattering of scalar particles by a black hole
}

\author{
M.Yu.Kuchiev圈 and V.V.Flambaum ${ }^{1,2}$ 讨 \\ 1 School of Physics, University of New South Wales, Sydney 2052, Australia and \\ 2 Institute for Advanced Study, Einstein drive, Princeton, NJ 08540, USA
}

(Dated: September 1, 2018)

\begin{abstract}
The absorption cross section for scalar particle impact on a Schwarzschild black hole is found. The process is dominated by two physical phenomena. One of them is the well-known greybody factor that arises from the energy-dependent potential barrier outside the horizon that filters the incoming and outgoing waves. The other is related to the reflection of particles on the horizon (Kuchiev 2003). This latter effect strongly diminishes the cross section for low energies, forcing it to vanish in the infrared limit. It is argued that this is a general property, the absorption cross section vanishes in the infrared limit for scattering of particles of arbitrary spin.
\end{abstract}

PACS numbers: 04.70.Dy, 04.20.Gz

\section{INTRODUCTION}

This work presents new qualitative features for scattering of particles by black holes. Interest in the scattering problem was first inspired long ago by the discovery of the Penrose process 1], which allows an impact particle to bring energy out of the Kerr black hole. After the works of Zel'dovich [2] and Misner [3] it became clear that the energy extraction from the Kerr black hole can be described in terms of superradiant scattering. The corresponding amplification factor was calculated numerically by Press and Teukolsky [4, 5] and analytically by Starobinsky [6] for the scalar field and Starobinsky and Churilov 7] for electromagnetic and gravitational waves. Independently, in parallel with this line of research, Unruh [8] found the absorption cross section for scalar and fermion particles scattered off a Schwarzschild black hole. The results of these and related works are well known, being summarized in books [9, 10, 11] that provide also further references on the subject.

An important qualitative feature of the scattering problem is related to the well-known greybody factors that arise from energy-dependent potential barriers outside the horizon, which filter the incoming and outgoing waves producing a strong impact on the cross section. In particular, they make the absorption cross sections finite, proportional to the event horizon area in the infrared region [12]. The greybody factors also manifest themselves in the Hawking radiation process 13, 14] filtering the initially blackbody spectrum emanating from the horizon. Refs. 15] describe a number of different aspects relevant to the greybody effect. Similarly, the potential barriers manifest themselves in the effect of gravitational lensing (for theory and references see the book [16]) that, in particular, can be caused by strong bending of light in the

\footnotetext{
*Email kuchiev@newt.phys.unsw.edu.au

${ }^{\dagger}$ Email flambaum@newt.phys.unsw.edu.au
}

vicinity of $r=(3 / 2) r_{g}>r_{g}[17]$.

The necessity to take another look at the scattering problem is prompted by Refs. [18, 19, 20] that claim that the horizon has a new unexpected property: it is able to partially reflect the incoming and outgoing waves. In other words, the events that take place on the horizon mix the incoming and outgoing waves. In simple physical terms this means that a particle approaching the vicinity of the horizon can bounce back, into the outside world. We will call this property the reflection from the horizon $(\mathrm{RH})$. The effect, which is strong for low energy particles, has a purely quantum origin, classically the particle penetrates the horizon smoothly.

The fact that the $\mathrm{RH}$ takes place strictly on the horizon distinguishes it from the greybody effect that happens outside of the horizon. This difference becomes particularly prominent for low energies of the incoming particle, when the greybody effect manifests itself at very large distances, much larger than the radius of the horizon. Another distinctive feature of the $\mathrm{RH}$ is its universal nature. The probability that a particle is reflected from the horizon is governed by only two parameters, the Hawking temperature and the energy of the incoming particle [24], being independent of the particle spin. This is different from the greybody effect that strongly depends on the spin of a particle. The fact that there appears the Hawking temperature is not coincidental, the $\mathrm{RH}$ can be used for alternative derivation of the Hawking radiation effect. (Reversing this argument, one can claim that the Hawking radiation supports the validity of the $\mathrm{RH}$.)

The existence of the RH should strongly manifest itself in scattering, making it necessary to reexamine the scattering abilities of black holes. This paper addresses this problem considering scattering of scalar particles by the Schwarzschild black hole. The problem is solved analytically for low impact energies and numerically for arbitrary energies. The analysis presented reveals that the $\mathrm{RH}$ reduces the absorption cross section. In particular, it forces the absorption cross section to vanish in the infrared regime $\sigma_{\text {abs }} \propto \varepsilon$ when $\varepsilon \ll \hbar c / r_{g} m>0$. It is ar- 
gued that this is a general feature, the absorption cross section vanishes in the infrared region for scattering of any particle off an arbitrary black hole.

\section{MODIFICATION OF THE $S$-MATRIX DUE TO REFLECTION FROM HORIZON}

Consider the scalar field $\phi(x)$ in the vicinity of the conventional Schwarzschild black hole with the metric

$$
d s^{2}=-\left(1-\frac{1}{r}\right) d t^{2}+\frac{d r^{2}}{1-1 / r}+r^{2} d \Omega^{2},
$$

where $d \Omega^{2}=d \theta^{2}+\sin ^{2} \theta d \varphi^{2}$. The relativistic units $\hbar=c=1$ are used and supplemented by the condition $2 G M=1$ on the gravitational constant $G$ and the mass of the black hole $M$, the Schwarzschild radius in these units reads $r_{g} \equiv 2 G M=1$. The Klein-Gordon equation $-\partial_{\mu}\left(\sqrt{-g} g^{\mu \nu} \partial_{\nu} \phi\right)=\sqrt{-g} m^{2} \phi$ for the field $\phi(x)$ in the Schwarzschild metric allows the separation of variables $\phi(x)=\exp (-i \varepsilon t) Y_{l m}(\theta, \varphi) \phi_{l}(r)$, where $\varepsilon, l, m$ are the energy, momentum and its projection, while $\phi_{l}(r)$ is a radial function that satisfies

$$
\begin{aligned}
& \phi_{l}^{\prime \prime}+\left(\frac{1}{r}+\frac{1}{r-1}\right) \phi_{l}^{\prime} \\
& +\left(p^{2}+\frac{\varepsilon^{2}+p^{2}}{r-1}+\frac{\varepsilon^{2}}{(r-1)^{2}}-\frac{l(l+1)}{r(r-1)}\right) \phi_{l}=0 .
\end{aligned}
$$

Here $p$ is the momentum at infinity. Let us describe with the help of Eq.(2.2) the scattering of the scalar particle by the black hole. At large radiuses $r \gg 1$ Eq.(2.2) reduces to a Coulomb-type equation with the effective Coulomb charge $Z=\left(\varepsilon^{2}+p^{2}\right) / 2=\varepsilon^{2}\left(1+v^{2}\right) / 2$, where $v$ is the velocity of the impact particle at infinity [25]. Therefore at large distances the solution can be presented as

$$
\phi_{l}(r) \rightarrow \frac{1}{r}\left(A_{l} \exp (i z)+B_{l} \exp (-i z)\right)
$$

where $z=p r+\nu \ln (2 p r)+\delta_{l}^{(C)}+l \pi / 2$ and

$$
\delta_{l}^{(C)}=\arg \Gamma(l+1-i \nu)
$$

is the Coulomb phase. Here $\nu=Z / p=v \varepsilon\left(1+1 / v^{2}\right) / 2$ is the conventional Coulomb parameter. Clearly the two terms in Eq. 2.3. describe the incoming and outgoing waves. The scattering properties can be expressed via the $S$-matrix that can be written as the ratio of the coefficients in front of the incoming and outgoing waves [21] that, accordingly, equals

$$
S_{l}=(-1)^{l+1} \frac{A_{l}}{B_{l}} \exp \left(2 i \delta_{l}^{(C)}\right) .
$$

The event horizon $r=1$ is a regular singular point of Eq.(2.2). In its vicinity the solution can be presented as $\phi_{l} \approx \exp (\mp i \varepsilon \ln (r-1))$. Here the waves with signs minus and plus describe the incoming and outgoing waves respectively. Since absorption of particles by the black hole is considered, it appeared appropriate to discard the outgoing wave, imposing the condition $\phi_{l}(r) \rightarrow \exp (\mp i \varepsilon \ln (r-1))$ on the horizon $r \rightarrow 1$, see Ref. [8]. However, recent Refs. [18, 19, 20] argue that this condition should be modified. The wave function describing the incoming particle should necessarily include an admixture of the outgoing wave

$$
\phi_{l}(r) \rightarrow \exp [-i \varepsilon \ln (r-1)]+\mathcal{R} \exp [i \varepsilon \ln (r-1)] .
$$

These works interpreted this boundary condition as a statement that the event horizon is able to partially reflect particles. The absolute value of the reflection coefficient $\mathcal{R}$ found in [18, 19, 20] reads

$$
|\mathcal{R}|=\exp [-\varepsilon /(2 T)],
$$

where $T$ is the Hawking temperature, $T=1 / 4 \pi$ for the Schwarzschild black hole. The origin and physical meaning of the $\mathrm{RH}$ were discussed in detail in the mentioned works. Here only brief comments are appropriate. Ref. 18] derives Eq. (2.6) from the general symmetry properties of the Schwarzschild geometry. The two disconnected areas that describe the outer region (areas I and III on the Kruskal plane [22], see [23]) are physically identical. Therefore there is a discrete symmetry that relates values of the wave function in these two regions. It is shown in [18] that the incoming wave by itself, i.e. the first term in Eq.(2.6), cannot satisfy this symmetry condition, whereas a linear combination of the incoming and outgoing waves complies with it, provided the reflection coefficient obeys Eq. (2.7). Another way to derive this result was suggested in [19]. We will discuss and use it below in order to find the phase of the reflection coefficient (proving it to be zero for low energy particles).

Let us find the relation between the $S$-matrix and the reflection coefficient $\mathcal{R}$. Since the latter decreases exponentially with energy we will consider first the low energy region $\varepsilon \ll 1$, where the $\mathrm{RH}$ is prominent, restricting our attention to the most important for this region case $l=0$ and denoting the s-wave as $\phi(r) \equiv \phi_{0}(r)$.

Following the approach of Ref. 8] consider three regions of distances. Region 1 we choose in the vicinity of the horizon $r \rightarrow 1$. The wave function here is given in Eq. (2.6). As region 2 we take those "intermediate" distances $1<r<\infty$, where one can neglect the low energy and momentum in Eq.(2.2). The solution of thus simplified equation can be written as

$$
\phi(r)=\alpha \ln \frac{r-1}{r}+\beta .
$$

Compare now Eq.(2.6) with (2.8) in the region of distances close, but not very close to the horizon $r-1 \ll 1$, i.e. the region where one can expand the incoming and outgoing waves in Eq. (2.6) $\exp [\mp i \varepsilon \ln (r-1)] \simeq$ $1 \mp i \varepsilon \ln (r-1)$ and use simultaneously the asymptotic 
relation $\ln [(r-1) / r] \simeq \ln (r-1)$ in Eq. (2.8). This procedure allows us to find the coefficients in Eq. (2.8)

$$
\alpha=-i \varepsilon(1-\mathcal{R}), \quad \beta=1+\mathcal{R} .
$$

Consider now region 3 , the region of large separations $r \gg 1$. The wave function behavior here is governed by the effective Coulomb problem. Introducing the regular $F(r)$ and singular $G(r)$ solutions of the Coulomb problem, see 21], one can present the wave function here as their linear combination

$$
\phi(r)=\frac{1}{r}(a F(r)+b G(r)) .
$$

Taking $r$ reasonably large one makes Eqs.(2.8) and (2.10) valid simultaneously. Eqs.(2.8), (2.9) give

$$
\phi(r) \simeq 1+\mathcal{R}+i \varepsilon(1-\mathcal{R}) \frac{1}{r} .
$$

On the other hand, since $\varepsilon$ is low we can assume that $p r \ll 1$ and use the asymptotic relations for small distances in the wave functions of the Coulomb problem

$$
F(r) \simeq C p r, \quad G \simeq 1 / C,
$$

see 21], where

$$
C^{2}=\frac{2 \pi \nu}{1-\exp (-2 \pi \nu)} .
$$

Eq.(2.10) then gives

$$
\phi(r) \simeq a p C+\frac{b}{C r} .
$$

Comparing Eqs.(2.11), (2.14) we find the s-wave coefficients in Eq. 2.10)

$$
a=\frac{1+\mathcal{R}}{p C}, \quad b=i \varepsilon C(1-\mathcal{R})
$$

Thus Eqs. 2.15), (2.10) define the behavior of the wave function at large distances. In the asymptotic region $r \rightarrow \infty$ we can use the known formulas, see [21], for the Coulomb functions

$$
F(r) \simeq \sin z, \quad G(r) \simeq \cos z,
$$

where $z=p r+\nu \ln 2 p r+\delta_{0}^{(\mathrm{C})}$, that allow us to present Eq.(2.10) in an asymptotic form (2.3). As a result we find the coefficients $A_{0}, B_{0}$ in the latter

$$
\begin{aligned}
& A_{0}=\frac{b-i a}{2 i}=\frac{1}{2 i p C}\left[1+\mathcal{R}-p \varepsilon C^{2}(1-\mathcal{R})\right] \\
& B_{0}=\frac{b+i a}{2 i}=\frac{-1}{2 i p C}\left[1+\mathcal{R}+p \varepsilon C^{2}(1-\mathcal{R})\right] .
\end{aligned}
$$

The corresponding $S$-matrix Eq.(2.5) for the s-wave reads

$$
S_{0}=\frac{1+\mathcal{R}-v \varepsilon^{2} C^{2}(1-\mathcal{R})}{1+\mathcal{R}+v \varepsilon^{2} C^{2}(1-\mathcal{R})} \exp \left(2 i \delta_{0}^{(\mathrm{C})}\right) .
$$

The factor $\mathcal{R}$ in this formula arises from the RH. If one wishes to neglect this phenomenon, one can put $\mathcal{R}=0$ in Eq.(2.6) and, correspondingly, in the $S$-matrix (2.18). Then Eq.(2.18) reproduces the results of [8], see discussion of Eq. (4.7) below. The factors $v \varepsilon^{2} C^{2}$ in the $S$-matrix originate from the greybody effect, they are present even in the $\mathcal{R}=0$ approximation. They arise due to those events that take place at large separations $r \sim 1 / Z=2 /\left[v^{2} \varepsilon^{2}\left(1+1 / v^{2}\right)\right] \gg 1$. This is in contrast with the $\mathrm{RH}$, which happens strictly at the horizon $r=1$. Thus Eq.(2.18) accounts for both the greybody factor and the effect of the $\mathrm{RH}$.

Using conventional expression for the inelastic cross section, see e. g. 21], we find from Eq.(2.18) the absorption cross section in the s-wave that dominates the process in the low energy limit.

$$
\begin{aligned}
\sigma_{\text {abs }} & =\frac{\pi}{p^{2}}\left(1-\left|S_{0}\right|^{2}\right)=\frac{4 \pi \xi C^{2}}{v\left(1+\xi v \varepsilon^{2} C^{2}\right)^{2}}, \\
\xi & =\frac{1-R}{1+R} .
\end{aligned}
$$

\section{REFLECTION COEFFICIENT OF THE HORIZON}

The absolute value of the reflection coefficient $\mathcal{R}$ is given in Eq.(2.7). In order to find its phase we follow the approach of Ref. 20]. Consider first the incoming wave in the outside region in the close vicinity of the horizon, $\phi_{\text {in }}(r)=\exp [-i \varepsilon \ln (r-1)]$ for $r \rightarrow 1, r>1$. Continue it into the interior region $r<1$ using an analytical continuation over the variable $r$ into the lower semiplane of the complex plane $r$. This procedure allows one to find the incoming wave in the interior region in the vicinity of the horizon, $r \rightarrow 1, r<1$,

$$
\phi_{\text {in }}(r)=|\mathcal{R}|^{1 / 2} \exp [-i \varepsilon \ln (1-r)] .
$$

It is suppressed compared to the outside region by a factor $|\mathcal{R}|^{1 / 2}=\exp (-\pi \varepsilon)$. Continue now the incoming wave further into the interior region $r<1$ using the differential equation (2.2). In the vicinity of the origin $r \rightarrow 0$ the equation simplifies, its solution here can be presented as

$$
\phi_{\text {in }}(r)=u \ln r+v,
$$

where $u, v$ are constants defined below. We assume that the total wave function should satisfy the conventional regular condition $\phi(r) \rightarrow$ const at the origin. Since the incoming wave by itself exhibits a singular $\propto \ln r$ behavior, there should exist the outgoing wave that compensates the singularity in the vicinity of the origin. Thus we find the outgoing wave in the vicinity of the origin. Repeating now the arguments in the reverse order, we take this outgoing wave and continue it towards the horizon using the differential equation; then continue it over the horizon using the analytical continuation via the lower 
semiplane of the complex $r$-plane. As a result there appears the outgoing wave in the outside region described by the second term in Eq.(2.6). Eq.(2.7) follows from the fact that the horizon is crossed twice, first by the incoming wave and then by the outgoing wave. Each crossing gives the suppression factor $|\mathcal{R}|^{1 / 2}$ that combine to produce $|\mathcal{R}|$ in (2.7). The method of crossing of the horizon used in this derivation relies on the analytical properties of the wave function that are related to the fundamental causality principle, which makes the method reliable.

Consider now the incoming wave in the inside region. Its behavior in the vicinity of the horizon and at the origin is defined by Eqs. (3.1) and (3.2). Take the "intermediate" region $0<r<1$, defined in such a way that inside this region one can neglect the small terms proportional to $\varepsilon, p$ in Eq.(2.2). The solution in this region reads

$$
\phi_{\text {in }}(r)=\alpha^{\prime} \ln \frac{1-r}{r}+\beta^{\prime}
$$

Consider now the region close, but not very close to the horizon $r \approx 1$, where Eqs. 3.1) and (3.3) are both valid. Expand Eq. (3.1) in powers of $\varepsilon \ln (1-r), \phi(r) \simeq$ $|\mathcal{R}|^{1 / 2}[1-i \varepsilon \ln (r-1)]$ and rewrite Eq.(3.3) for this region as $\alpha^{\prime} \ln (1-r)+\beta^{\prime}$. This procedure gives the coefficients in Eq. 3.3

$$
\alpha^{\prime}=-i|\mathcal{R}|^{1 / 2} \varepsilon, \quad \beta^{\prime}=|\mathcal{R}|^{1 / 2}
$$

Consider now the region close, but not very close to the origin $r \approx 0$, where Eqs. (3.2) and (3.3) are valid simultaneously. Rewriting (3.3) in this region as $\phi_{\text {in }}(r)=$ $-\alpha^{\prime} \ln r+\beta^{\prime}$, we find the coefficients

$$
u=-\alpha^{\prime}=i|\mathcal{R}|^{1 / 2} \varepsilon, \quad v=\beta^{\prime}=|\mathcal{R}|^{1 / 2},
$$

which define the behavior of the incoming wave at the origin in Eq.(3.2). It is important that the coefficient $u$ in front of the singular term in Eq. 3.2 is imaginary, while $v$ that describes the regular part of the solution is real. To see consequences of this fact let us take the outgoing wave $\phi_{\text {out }}(r)$ that behaves at the horizon $r \rightarrow 1$ as

$$
\phi_{\text {out }}(r)=|\mathcal{R}|^{1 / 2} \exp [i \varepsilon \ln (1-r)] .
$$

According to Eq. 3.1 this outgoing wave is simply a complex conjugate of the incoming wave. From Eq.(3.2) we find that $\phi_{\text {out }}(r) \rightarrow u^{*} \ln r+v^{*}$ when $r \rightarrow 0$. Using Eq.(3.5) we conclude that the wave function defined as

$$
\phi(r)=\phi_{\text {in }}(r)+\phi_{\text {out }}(r)
$$

is regular at the origin, $\phi(r) \rightarrow 2 v=|\mathcal{R}|^{1 / 2}$ when $r \rightarrow 0$. Using the analytical continuation described above for crossing of the event horizon, we find that in the outside region $r>1$ the wave function defined in Eq. (3.7) coincides with the wave function in Eq.(2.7). The fact that the coefficients in front of the incoming and outgoing waves in Eq. (3.7) are identical (unity) permits one to find the reflection coefficient [19]

$$
\mathcal{R}=\exp [-\varepsilon /(2 T)]=\exp (-2 \pi \varepsilon) .
$$

The absolute value of the reflection coefficient found here agrees with Eq.(2.7) (though Eq.(3.8) is derived in the low energy limit $\varepsilon \ll 1$, while Eq.(2.7) remains valid for arbitrary energies; compare [18, 20] for alternative methods of derivation of Eq.(2.7) .

\section{ABSORPTION CROSS-SECTION}

We can now substitute the reflection coefficient from (3.8) into the $S$-matrix in Eq.(2.18). Expanding where possible the result in powers of $\varepsilon$ we find

$$
\begin{aligned}
S_{0} & =\frac{1-v \varepsilon^{2} C^{2} \tanh (\pi \varepsilon)}{1+v \varepsilon^{2} C^{2} \tanh (\pi \varepsilon)} \exp \left(2 i \delta_{0}^{(\mathrm{C})}\right) \\
& \simeq\left(1-2 \pi v \varepsilon^{3} C^{2}\right) \exp \left(2 i \delta_{0}^{(\mathrm{C})}\right) .
\end{aligned}
$$

Correspondingly, Eq.2.19) gives the absorption cross section

$$
\begin{aligned}
\sigma_{\mathrm{abs}} & =\frac{4 \pi C^{2} \tanh (\pi \varepsilon)}{v\left(1+v \varepsilon^{2} C^{2} \tanh (\pi \varepsilon)\right)^{2}} \\
& \simeq \frac{4 \pi^{2} \varepsilon C^{2}}{v}=\frac{4 \pi^{3} \varepsilon^{2}\left(1+1 / v^{2}\right)}{1-\exp \left[-\pi v \varepsilon\left(1+1 / v^{2}\right)\right]}
\end{aligned}
$$

where Eq.(2.13) was used. The last equation is presented for $\pi \varepsilon \ll 1$. For massless particles (or any particle with $\pi \varepsilon / v \ll 1)$

$$
\sigma_{\mathrm{abs}} \simeq 4 \pi^{2} \varepsilon \equiv \frac{4 \pi^{2} \varepsilon r_{g}^{3}}{\hbar c}
$$

(the last expression in absolute units) the cross section vanishes in the infrared limit $\varepsilon \rightarrow 0$. In contrast, for slow massive particles $v \rightarrow 0(\varepsilon \ll 1, \varepsilon / v \gg 1)$ the cross section diverges as

$$
\sigma_{\mathrm{abs}}=\frac{4 \pi^{3} m^{2}}{v^{2}} \equiv \frac{4 \pi^{3} m^{2} c^{4} r_{g}^{4}}{\hbar^{2} v^{2}}
$$

(absolute units). Compare these results with the previously known ones. If one neglects the $\mathrm{RH}$ effect, putting $\mathcal{R}=0$ in the $S$-matrix (2.5) and substituting the latter into Eq.(2.19) then one finds the cross section

$$
\sigma_{\mathrm{abs}}^{(\mathcal{R}=0)}=\frac{4 \pi^{2} \varepsilon\left(1+1 / v^{2}\right)}{1-\exp \left[-\pi v \varepsilon\left(1+1 / v^{2}\right)\right]},
$$

that was first derived by Unruh [8]. Comparing Eqs. (4.4), 4.7) we see that for low energy particles the effect of the $\mathrm{RH}$ reduces the cross section by a factor of $\pi \varepsilon \equiv \pi \varepsilon r_{g} / \hbar c \ll 1$, which is particularly important for 
massless particles in the infrared region where Eq.(4.7) predicts the constant cross section

$$
\sigma_{\mathrm{abs}}=4 \pi r_{g}^{2}
$$

(absolute units), which differs qualitatively from Eq.4.5. that describes the vanishing cross section. Let us repeat that the arguments of [18, 19, 20], some of which are partially reproduced above, indicate that the horizon possesses the reflective property that inevitably leads to Eq. (4.4).

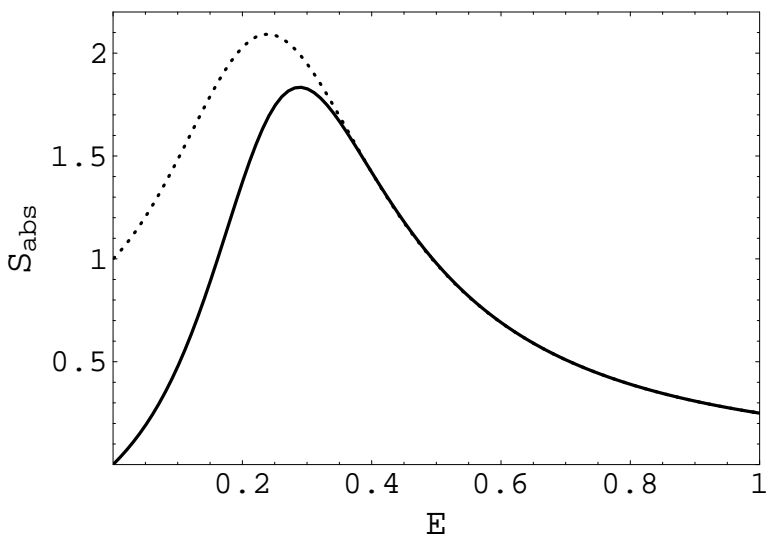

FIG. 1: The absorption cross section $S_{\text {abs }}$ (in units of the horizon area, $\left.S_{\text {abs }} \equiv \sigma_{\text {abs }} /\left(4 \pi r_{g}^{2}\right)\right)$ for the s-wave scattering $(l=0)$ of massless scalar particles by the Schwarzschild black hole versus the energy of the particle $E$ (dimensional units $\left.E \equiv \varepsilon r_{g} / \hbar c\right)$. Solid line - the reflection on the horizon is taken into account, for low energy Eqs. (4.4), (4.5) of this work are valid. Dashed line - the reflection by the horizon is not taken into account, at low energy this cross section agrees with Eqs. 4.7, (4.8) derived by Unruh 8].

The approach described above was also implemented numerically. The differential equation (2.2) was solved separately in the region $0<r<\infty$, using the discussed analytical continuation over the event horizon to match solutions in the inside and outside areas. (The regular at the origin solution is presented as a linear combination of the incoming and outgoing waves, each wave is continued over the horizon using the analytical continuation into the lower semiplane of the complex $r$-plane.) The numerical method permits one to find the cross section for arbitrary, not necessarily low energies. Generally speaking, such analysis needs that higher multipoles of the wave function be included. However, for energies $\varepsilon \leq 1$ the s-wave alone should give reasonable results. Fig. 1 presents our results for the s-wave absorption cross section of massless scalar particles. For $\varepsilon \ll 1$ they reproduce Eq.(4.4). Fig. 1 presents also results of similar calculations for the cross section when one neglects the reflective ability of the horizon, putting $\mathcal{R}=0$ in Eq. 2.6) (which allows one to formulate the problem entirely in the outside region). Fig. 11 shows that the reflective ability of the horizon strongly diminishes the cross section in the low-energy region producing smaller impact for higher energies. This fact agrees qualitatively with Eq.2.7 that states that the reflection coefficient diminishes exponentially with the energy increase.

\section{DISCUSSION}

As is known a classical particle can not reach the horizon during a finite interval of time in the reference frame of the external observer described by the metric Eq.(2.1). In this (restrictive) sense, the horizon represents an impenetrable barrier for the incoming particle. In the quantum process a role of the horizon is more subtle. One can look at it considering the conserving current corresponding to the Klein-Gordon equation $-\partial_{\mu}\left(\sqrt{-g} g^{\mu \nu} \partial_{\nu} \phi\right)=\sqrt{-g} m^{2} \phi$ :

$$
j^{\mu}=\frac{1}{2 i} \sqrt{-g} g^{\mu \nu}\left(\phi^{*} \partial_{\nu} \phi-\phi \partial_{\nu} \phi^{*}\right)
$$

Near the horizon for $r>1$ the incoming wave function $\phi_{\text {in }}(r)=\exp [-i \varepsilon \ln (r-1)]$ gives the following current components

$$
\begin{aligned}
& j^{0}=\varepsilon \frac{r}{r-1} \sqrt{-g}, \\
& j^{r}=-\varepsilon \frac{1}{r} \sqrt{-g} .
\end{aligned}
$$

We see that for the considered stationary solution the radial current does not have any singularity at the horizon $r=1$. Zero velocity of the particle (in the reference frame of the external observer) is compensated by the infinite density.

Consider now the current near horizon inside the black hole for the incoming wave $\phi_{\text {in }}(r)=\sqrt{|\mathcal{R}|} \exp [-i \varepsilon \ln (1-$ $r)]$

$$
\begin{aligned}
j^{0} & =-\varepsilon|\mathcal{R}| \frac{r}{1-r} \sqrt{-g}, \\
j^{r} & =-\varepsilon|\mathcal{R}| \frac{1}{r} \sqrt{-g} .
\end{aligned}
$$

The radial current is still directed towards the center. However, the zero-th component, i. e. the probability density, becomes negative. A possible interpretation of this result can be related to a state of the "hole" that is produced inside. We call here by the hole a negative-energy state of the scalar field. Another popular way to call this state is to dub it as the "antiparticle with negative energy". (The hole considered here should not, of course, be confused with the black hole itself.) Propagation of the hole from the origin $r=0$ to the horizon creates the radial current towards the origin. Similarly we can consider the outgoing wave inside $\phi_{\text {out }}(r)=\sqrt{|\mathcal{R}|} \exp [i \varepsilon \ln (1-r)]$, which describes the current directed from the origin to the horizon. In this wave the hole is produced on the horizon $r=1$ and moves towards the origin $r=0$. The fact that the wave function inside Eq.(3.7) includes both the incoming and outgoing 
waves shows that the state of the hole inside produces zero radial current. To finish the argument, the outgoing wave in the outside region $\phi_{\text {out }}(r)=|\mathcal{R}| \exp [i \varepsilon \ln (r-1)]$ obviously describes the outgoing particle that moves from the horizon $r=1$ to infinity.

Combining all peaces together, one can say that the horizon is responsible for the creation of a particle-hole pair and for the annihilation of another particle-hole pair. It creates the incoming hole that moves inside and the outgoing particle that moves from the horizon to infinity. The hole created on the horizon moves towards the origin, is reflected there back towards the horizon, where its encounter with the incoming particle results in their mutual annihilation. The net result is the outgoing particle outside, which is exactly the RH.

Here we find again a certain similarity between the Hawking radiation and the $\mathrm{RH}$. In both phenomena the horizon creates the particle-hole pair with the probability that depends exponentially on $\varepsilon / T$. There is also a distinction. In the $\mathrm{RH}$ phenomenon a creation of one pair is accompanied by annihilation of another pair on the horizon, which shows that it is a more complicated phenomenon compared to the Hawking radiation.

\section{CONCLUSION}

We discussed above two effects that combine together to make the absorption cross section small at low energies. The first one is the known greybody effect that takes place outside of the event horizon, at the radius of the Coulomb zone that is large, $r \gg r_{g}$, for low energies. In the Coulomb zone the semiclassical approximation is violated making possible the reflection of the incoming wave. This reflection reduces the absorption cross section, making it finite, proportional to the area of the horizon in the infrared limit.

Another opportunity for the reflection is presented by the $\mathrm{RH}$ effect that takes place strictly on the horizon. It reduces the cross section further, forcing it to vanish in the infrared limit. Importantly, one can expect that this is a general property of the cross section valid for the scattering of particles of arbitrary spins. This expectation is based on the fact that Refs. [18, 19, 20] appeal to the semiclassical nature of the radial wave function in the vicinity of the horizon, being valid for the scattering of particles of arbitrary spins. There is a subtlety here, to verify this expectation one needs to prove that the phase of the reflection coefficient turns either 0 , or $\pi$ (which makes $\xi$ in Eq. (2.20) either 0, or $\infty$ ), in the infrared region for arbitrary spins. This is a very plausible opportunity, but, strictly speaking, the present work does not discuss it. It is also plausible that a similar more general statement can be formulated for scattering of particles of any charge and spin off a general Kerr-Newman black hole. The reflection coefficient in this case 20] can be large, $|\mathcal{R}|=1$ for particular values of energy, charge and spin of the impact particle, which makes the reflection perfect and absorption impossible (though, again, to prove this result the particular phase conditions should be verified). Several last remarks provide possible ways for further study.

In conclusion, it is shown that the reflection on the horizon strongly influences the absorption cross section of a black hole, forcing it to vanish in the infrared limit.

This work was supported by the Australian Research Council. V.F. is grateful to the Institute for Advanced Study and Monell foundation for hospitality and support.
[1] R. Penrose, Revista. Nuovo. Cim. 1, 252 (1969).

[2] Ya. B. Zel'dovich, Zh. Eksp. Teor. Fiz. Pis'ma Red. 14, 270 (1970) [JETP Lett. 14, 180 (1971)]; Zh. Eksp. Teor. Fiz. 62, 2076 (1972) [Sov.Phys.JETP 35, 1085 (1972)].

[3] C. W. Misner, Bull. Am. Phys. Soc. 17, 472 (1972).

[4] W.H. Press, S. A. Teukolsky, Nature (London) 238, 211 (1972);

[5] W.H. Press and S. A. Teukolsky, Astrophys. J. 141, 443 (1974).

[6] A. A. Starobinsky, Zh. Eksp. Teor. Fiz. 64, 48 (1973) [Sov.Phys.JETP 37, 28 (1973)].

[7] A. A. Starobinsky and C. M. Churilov, Zh. Eksp. Teor. Fiz. 65, 3 (1973) [Sov.Phys.JETP 38, 1 (1974)].

[8] W. G. Unruh, Phys. Rev. D 14, 3251 (1976); thesis Princeton Univ., 1971 (unpublished) (available University Microfilms, Ann Arbor, Mich.).

[9] V. P. Frolov and I. D. Novikov, Black hole physics: basic concepts and new developments (1998) Dordrecht; Boston: Kluwer.

[10] S. Chandrasekhar, The Mathematical Theory of Black Holes (1992) New York: Oxford University Press.

[11] J.A.H. Futterman, F.A. Handler, and R.A. Matzner,
Scattering from black holes (1988) Cambridge; New York: Cambridge University Press.

[12] S. A. Kaplan, Zh. Eksp. Teor. Fiz. 19, 951 (1949); Ya. B. Zel'dovich and I. D. Novikov, Dokl. Acad. Nauk. 155, 1033 (1960).

[13] S. W. Hawking, Nature (London) 248, 30 (1974).

[14] S. W. Hawking, Commun. Math. Phys. 43, 199 (1975).

[15] J. D. Bekenstein, Phys. Rev. Lett. 70, 3680 (1993); C. F. E. Holzhey and F. Wilczek, Nucl.Phys. B380, 447 (1992); V. Balasubramanian and F. Larsen, Nucl. Phys. B495 206, (1997); J. M. Malcadena and A. Strominger, Phys. Rev. D55, 861 (1997); H.C. Rosu and M. Planat, Mod.Phys.Lett. A17 1377(2002); S. Lepe, F. Mndez, and J. Saavedra, Fermion scattering in a three dimensional extreme black hole background, hep-th/0302035

[16] Schneider P., Ehlers J., and Falco E. E., Gravitational lenses (1992) Springer-Verlag, Berlin.

[17] Virbhadra K. S. and Ellis G. F. R., Phys. Rev. D 62 (2000) 084003.

[18] M.Yu.Kuchiev, Reflection from black holes, gr-qc/0310008

[19] M.Yu.Kuchiev. Reflection from black holes and space- 
time topology, gr-qc/0310134 Europhysics Letters (accepted).

[20] M.Yu.Kuchiev. Reflection, radiation and interference for black holes gr-qc/0310051

[21] L. D. Landau and E. M. Lifshits, Quantum mechanics: non-relativistic theory (1977) New York: Pergamon.

[22] M. D. Kruskal, Phys. Rev. 119, 1743 (1960).

[23] C. W. Misner, K. S. Thorn, J. A. Wheeler, Gravitation
(San Fransisco: W. H. Freeman and Company 1973).

[24] This is true for the Schwarzschild case, see details in 20] that describes the $\mathrm{RH}$ for charged rotating black holes

[25] There is also a modification of the momentum due to the term $\varepsilon^{2} /(r-1)^{2}$ in Eq. (2.2), but one can neglect it in the low energy limit $\varepsilon \ll 1$. 